\title{
Discussion on Redesign of Image from Digital Printing Process
}

\author{
Mingying Luo ${ }^{1}$ \\ ${ }^{1}$ School of Architecture \& Artistic Design, University of Science and technology Liaoning, Anshan, Liaoning, \\ China \\ Correspondence: Mingying Luo, School of Architecture \& Artistic Design, University of Science and technology \\ Liaoning, Anshan 114051, Liaoning, China.
}

Received: June 9, 2017

Accepted: June 16, 2017

Online Published: June 27, 2017

doi:10.20849/ajsss.v2i2.163

URL: https://doi.org/10.20849/ajsss.v2i2.163

This is a project for teaching reform of provincial undergraduate courses in Liaoning Province in 2016.

\begin{abstract}
Digital printing is an indispensable link in the modern printing technology. It is the traditional pre-press process on the transition to digital technology in imaging technology, the digital printing technology. Digital printing expounds digital image process in the design means and methods and their influences on the printing quality from the angle of separation method, image processing method, etc..
\end{abstract}

Keywords: printing digitalization, image technology, prepress process, redesign

There are some differences between digital printing and printing digitalization. Digital printing mainly refers to the process of digital pre-press technology. Digital printing is the replication process which will copy the text information by digital control system through a technique or process directly to the production record medium and complete the process. Printing digitalization is the improvement of printing method. Digital printing is the progress of printing technology. Digital printing, like other emerging things, represents the progress of printing technology. On the other hand, it still needs to be perfected. The digital printing in the printing of diversification is imperative, especially in different printing fields, through the digital printing process, then designs the image and improves the printing quality, so as to meet the needs of different technologies on printing quality.

\section{The Origin of Digital Printing}

In the early nineteenth century, French artists Neps (J.N. Niepce) and Daguerre (L.J.M. Daguerre) began to study successful daguerreotype, start coating photographic layer on the metal plate, and obtained fine image on camera. Since then, with the camera technology, the technology can only shoot from the photographic plate wet plate photography monochrome line copy. Later, that technology evolved to shoot with network photography continuous tone, and then developed into color photography and photographic color separation method. (Feng, Ruiqian, 1984) Then, emerged the color image with complex photographic color separation and screening, film output etc. the prepress process, the color printing and copying technology. Therefore, in the development of modern printing, photography and printing have the indissoluble bound.

With the progress of technology, in the middle of 80s, the United States Apple company launched Macintosh series computer, laser printer output and produced to high definition and PageMakerl.0 typesetting software developed. The desktop publishing system DTP (Desktop Publishing), which uses computer technology, graphic layout and page description language as its main part, marks the arrival of the printing digital era (Wu, Jianjun, 2006).

After nearly 20 years of development, desktop publishing system has become a comprehensive application of graphic input, graphic production, layout, graphic output and network transmission, digital integration technology in the printing field. With Adobe Photoshop, Painter, Adobe Illustrator, CorelDraw software being introduced, which further narrows the relationship between design and printing, the designer is displayed and enters into a new space.

The so-called digital printing, is the product of the progress of the times, a non-digital manuscript by digital equipment into digital quadrochromatic computer which can operate. The two digital quadrochromatic gets or is produced by direct digital equipment. The former is the application of digital printing equipment, while the latter 
is the basic ability of modern designers, but also one of the digital printing. Digitized manuscript is designed to meet the DTP desktop publishing system control, and to achieve "WYSIWYG" digital prepress technology operation. Therefore, from different aspects to improve the printing quality, convenient designers redesign the prepress image and design designers achieve the dream of heart.

\section{What Is Redesign?}

In the traditional printing process, the color of the original document needs to be decomposed before the printing, and the corresponding plate is made by the color after the decomposition. After the superposition of various inks, the printing of the color, the text and the image can be realized, and the process is printing. In the process of prepress processing, the quality of color separation and the processing technology of color separation edition determine the quality of printing. As the process mainly depends on the photography technology, the redesign of the image has always been plagued and restricted by photography technology.

With the emergence of DTP desktop publishing system, digital manuscript greatly facilitates prepress graphic processing and redesign. The design is original in all kinds of digital graphics and image software under the effect of treatment on color image, after printing the image to achieve zoom, rotation, distortion, emergence, trapping and other means after treatment set design. The redesigned image, which can improve the appearance of the original image and change the quality of the printed material, is one of the main means to realize high quality printing.

\section{The Process of Image Redesign in Digital Printing Process}

In the process of digital printing, image redesign mainly refers to the technology of color separation in spot color printing. Since the advent of color printing, color printing technology, an amazing process, has always been plagued by the designer when displayed, resulting in large number of excellent works not exquisite reproduction. RGB color separation, color separation light due to constraints, the layout of printing color overlay proportion of balanced superposition from any colors in the printing process, will change the tone of the whole picture, not the true color reduction (Liu, Quanxiang, 2006). And spot color separation is the use of a variety of color separation technologies and techniques to obtain a variety of color separation printing plate combinations, according to the design of creative ideas to achieve a multi-color printing. Spot color printing process after local color, image changes, which enriches the printing color, improves the image authenticity and nature; spot color printing process after processing of color printing, can improve the quality of printing, and obtain novel patterns, so as to realize the design and printing. Therefore, in the process of digital printing, after digitizing the original manuscript, in the image processing software, it greatly facilitates the designer's redesign of graphics and images.

\subsection{Layer Color Separation Method}

The layers in the PS software platform, and, for the designer's understanding, is a piece of transparent paper. Tracing and drawing, no matter how to paint on the layer, will not affect the following layer, when the top layer can cover the following layer. Also, tracing and drawing can be changed according to the demand of the transparency of the coating and other settings. See below the layer, so that you can copy the other map content, and can also carry out according to the drawing of the layer. Each layer can separate color, copy and freely transform the processing. Finally, it can also be set to multiply and simulate the color overlay effect printing. Therefore, the layered rendering has strong flexibility. The layer function facilitates the design of spot color printing, and realizes the redesign of images from different paths (Figure 1).
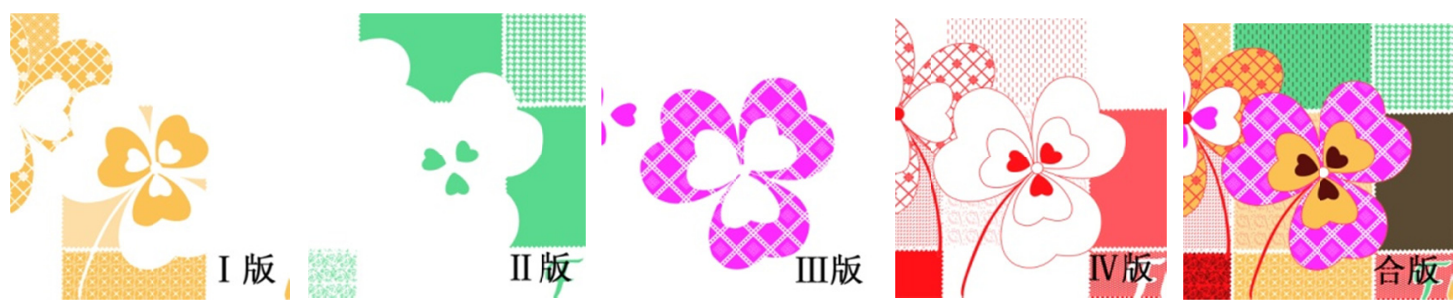

Figure 1. Fabric printing, color separation and pattern

\subsection{Color Separation Method}

Color selection is one of the application flexibility of PS software, and the constituency is mainly used to 
separate one or more parts of an image. Typically, in image processing, this is applicable not for the whole picture, but for some images or regions processing. Selecting the constituency means very flexible, frame selection and selection of lasso color range. Selection by different means can achieve any of different images with different color selection. Selection of the image or color can be copied, and adjust the free transform processing and preserved in a new layer. This function greatly facilitates the designer's application of natural material, especially the texture of natural materials, and realizes the recreation of reality and nature. Constituency color separation method, arbitrary selection function for printing color separation, image creation in design convenience (Figure 2).
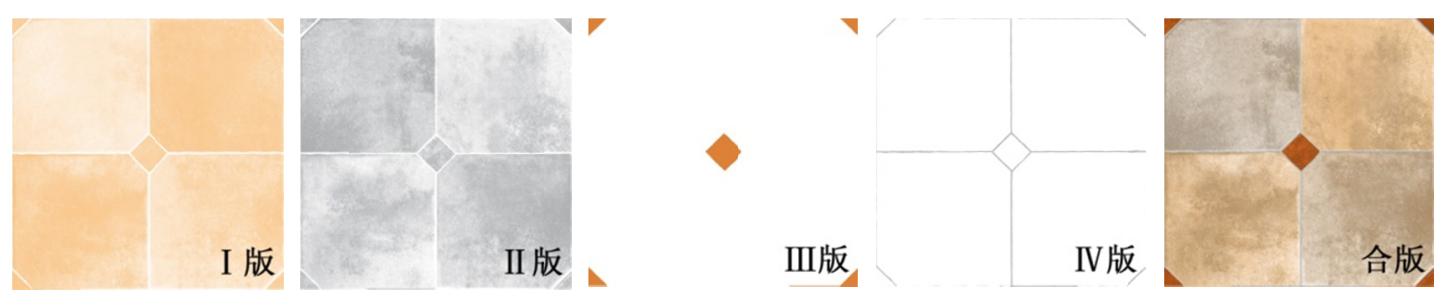

Figure 2. Plastic film printing, color separation and pattern

\subsection{Filter Color Separation Method}

Filter is the most abundant and unique tool in PS software. It can produce rich and colorful artistic effects. The so-called filter color separation is the use of filter function for image design talent performance. The filter in the PS software function which is very powerful, can magically transform the layer or image, and can be targeted to the performance of talent selection, so that it can create the image layer and the desired effect. The filter function has the ability to surpass the designer's intelligence, and magical changes provide designers with creative ideas. Therefore, in the process of image design, the designer should combine the features of the image, fully tap the filter function, display the color separation feature skillfully, and realize the perfect reproduction of the printing process (Figure 3).
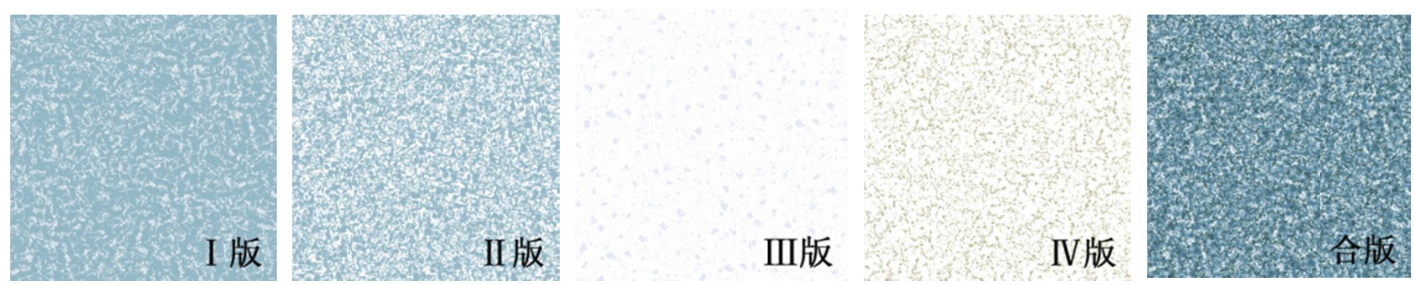

Figure 3. Printing, color separation and design of decorative materials

\section{4 channel Color Separation}

For PS software, the channel is to use special gray storage of image color information and spot color information. On the channel, graphics, editing, and filter processing can be performed to preserve image color information and to provide support for the preservation of constituencies. The color information recorded from beginning to end in the channel is closely related with your operation. The number of images containing different colors of the channel is not the same. Therefore, in the operation of desktop publishing system, especially in spot color printing, the channel creates convenient conditions for it. In the printing field, the channel can be understood as the spot color layer information, and the image redesign process can be realized by editing different channels. The channel type composite channel, color channel, spot color channel, single channel, Alpha channel, and the channel for spot color separation and image processing provide the ability to show (Figure 4). 

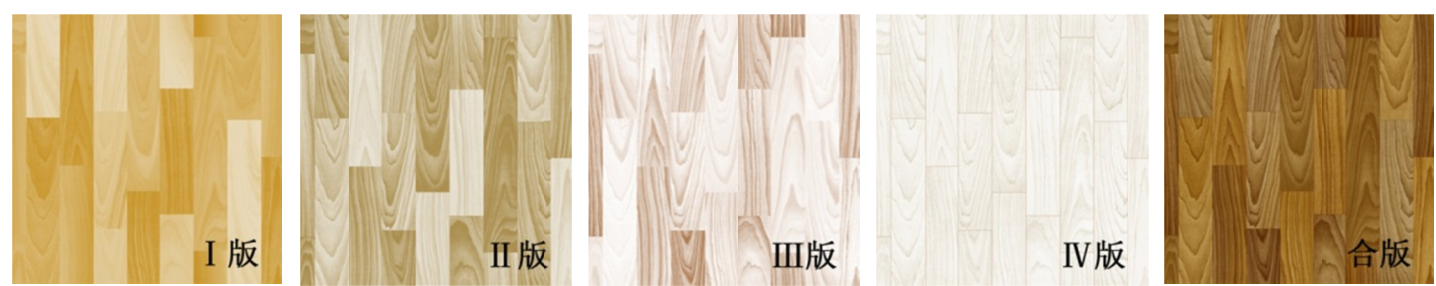

Figure 4. Composite floor printing color separation and pattern

\section{Epilogue}

With the progress of technology and the development of printing, from the beginning of woodblock printing, experienced photography era to today's digital technology era, and from the development of digital printing to printing digitalization, the development from printing to non-printing technology has marked the rapid progress of printing technology. Digital printing technology today not only affects the influence of image technology on printing quality, but also greatly facilitates the prepress process, to achieve "WYSIWYG" operating platform. At the same time, it also influences the prepress process flexibility and operability, and further realizes the design and technology of image.

\section{References}

Afterglow. (2008). Photoshop Image Processing. Shanghai: China Version of the Group.

Feng, R. (1984). Introduction to Printing Technology. Beijing: Printing Industry Press.

Liu, Q. (2006). Digital Printing Technology. Beijing: Printing Industry Press.

Wu, J. (2006). Printing Media Design. Beijing: China Architecture and Building Press.

\section{Copyrights}

Copyright for this article is retained by the author(s), with first publication rights granted to the journal.

This is an open-access article distributed under the terms and conditions of the Creative Commons Attribution license (http://creativecommons.org/licenses/by/4.0/). 